\title{
Purifying Electron Spectra from Noisy Pulses with Machine Learning Using Synthetic Hamilton Matrices
}

\author{
Sajal Kumar Giri, Ulf Saalmann, and Jan M. Rost \\ Max-Planck-Institut für Physik komplexer Systeme, Nöthnitzer Straße 38, 01187 Dresden, Germany
}

(Received 9 August 2019; accepted 18 February 2020; published 17 March 2020)

\begin{abstract}
Photoelectron spectra obtained with intense pulses generated by free-electron lasers through self-amplified spontaneous emission are intrinsically noisy and vary from shot to shot. We extract the purified spectrum, corresponding to a Fourier-limited pulse, with the help of a deep neural network. It is trained on a huge number of spectra, which was made possible by an extremely efficient propagation of the Schrödinger equation with synthetic Hamilton matrices and random realizations of fluctuating pulses. We show that the trained network is sufficiently generic such that it can purify atomic or molecular spectra, dominated by resonant two- or three-photon ionization, nonlinear processes which are particularly sensitive to pulse fluctuations. This is possible without training on those systems.
\end{abstract}

DOI: 10.1103/PhysRevLett.124.113201

Recent years have seen an avalanchelike increase of machine-learning applications in physics [1-3], which roughly fall into three categories: (a) applications within theory, e.g., for quantum information [1] or to elucidate intricate many-body properties [4], (b) within experiment to optimize experimental conditions, e.g., to characterize a free-electron laser (FEL) pulse [5], and (c) applications that condition learning algorithms theoretically with the goal to apply the trained model to experimental data. Our work falls in category (c). Although in principle far more general, we choose to be specific and apply the approach we develop to the purification of noisy photoelectron spectra as routinely obtained with self-amplified spontaneous emission (SASE) FELs operating in the desired frequency range.

Our goal is to train a deep neural network with sufficiently many noisy spectra and their pure counterparts, such that the trained network will be able to purify a noisy spectrum which is not contained in the training data, in particular an experimental one. With purification, we mean that upon feeding with a noisy photoelectron spectrum the network returns a reference spectrum that would be obtained if the target system would be driven by an ideal Gaussian laser pulse, which we call the reference pulse, cf. Fig. 1. This may seem straightforward. Yet, it is anything but trivial to generate a sufficient amount of suitable training data with an acceptable effort. This is in

Published by the American Physical Society under the terms of the Creative Commons Attribution 4.0 International license. Further distribution of this work must maintain attribution to the author(s) and the published article's title, journal citation, and DOI. Open access publication funded by the Max Planck Society. general the bottleneck for machine-learning applications in theory which requires new ways of thinking. In this vein, we introduce synthetic Hamilton matrices (SHMs). Synthetic means that we vary the matrix elements (here in a random fashion) about base values such that later on the trained network is able to purify real spectra from either experiment or theory. The SHMs are constructed to speed up the generation of training data and we also expect them to become useful for other dynamical problems for which neural networks must be trained. Since the SHMs cover a large range of possible systems we can afford to use for the base itself explicitly calculated photoionization dynamics in one dimension which is fast to compute and provides a suitable anchor point for the SHMs.

Setting up networks with SHMs.-To put our approach to a credible test we need (i) a physical process, which is sensitive to the pulse profile, (ii) a realistic way to model fluctuating pulses and we need to prepare a large set of spectra suitable for training the network. This involves (iii) a scheme to efficiently propagate millions of timedependent Schrödinger equations, (iv) a broad and uniform

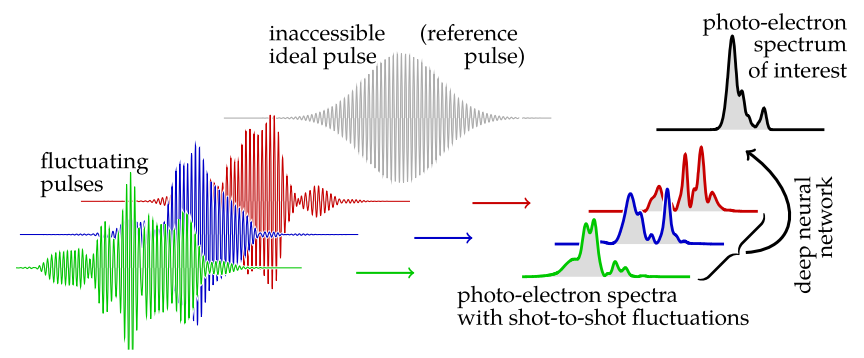

FIG. 1. Sketch of the problem: Photoelectron spectra from fluctuating pulses are purified using a deep neural network. 
sampling of the generated spectra, and (v) a trainable parametrization.

(i) As a physical process which is nonlinear in the driving light and therefore very sensitive to the intensity of the light pulse and hence its profile in time, we have chosen quasiresonant few-photon ionization. It can lead to multipeak structures in the photoelectron spectrum [6-10].

(ii) Fluctuating pulses from SASE FELs can be modeled by the so-called partial-coherence method [11,12], an experimentally verified method, which allows one to create ensembles of pulses $f_{l}(t)$ which differ through fluctuations while the ensemble average converges to a well defined pulse shape [13]. Those pulses have a characteristic duration $T$ and a coherence time $\tau$, we use $T=3$ fs and $\tau=1 / 2$ fs here. Apart from the intrinsic noise the pulses additionally jitter in their pulse energy. We normalize all pulses $f_{l}(t)$ to unit pulse energy. This is also possible in the experiment as pulse energies can be easily measured shot to shot with gas monitoring detectors [22].

(iii) In principle numerical codes are available to propagate the time-dependent Schrödinger equation (TDSE) for one active electron in a strong laser field and calculate the resulting photoelectron spectrum $P(E)$ [23-25]. However, the creation of a training data set from millions of pulses is prohibitively expensive, yet essential for successful deep learning.

To overcome this obstacle we work with Hamilton matrices whose construction is detailed in the Supplemental Material [13]. The new element, particularly formulated for the present context, is the generation of $n_{\text {mat }}$ Hamilton matrices with random energies $E_{\alpha}^{k}$, coupling matrix elements $V_{\alpha \beta}^{k}$, and field strengths $A_{k}$, corresponding to intensities (referring to the Fourier-limited pulse) in the range of $5 \times 10^{15}, \ldots, 5 \times 10^{16} \mathrm{~W} / \mathrm{cm}^{2}$. Furthermore, for each Hamilton matrix the coupling to the light is augmented by $n_{\text {pul }}$ noise realizations $f_{l}(t)$ with a central frequency of $21 \mathrm{eV}$ to arrive at

$$
\begin{aligned}
\mathbf{E}_{k} & =E_{\alpha}^{k} \delta_{\alpha \beta}, \quad \mathbf{V}_{k}=V_{\alpha \beta}^{k}, \\
\mathbf{H}_{k l}(t) & =\mathbf{E}_{k}+A_{k} f_{l}(t) \mathbf{V}_{k},
\end{aligned}
$$

whereby $k=1, \ldots, n_{\text {mat }}$ and $l=1, \ldots, n_{\text {pul }}$. Boldface symbols in Eqs. (1) describe matrices in terms of field-free states. It is only through these synthetic Hamilton matrices that we are able to create a sufficient number of nontrivial training data. The matrices have been derived varying a 1D Hamilton operator (our base system), but since the energies $\mathbf{E}_{k}$ and the coupling matrix elements $\mathbf{V}_{k}$ are chosen randomly, these SHMs can purify real (3D) spectra, as we will see subsequently.

(iv) We have to create a set of spectra for training, validation, and testing which should cover to a large extent the domain of realizable spectra. This step is crucial and most expensive numerically, particularly when compared to the (modest) resources needed to set up and train the network. To cover the domain of realizable spectra uniformly, we calculate first $4 \times 10^{4}$ reference spectra [26]. Among those we select the $n_{\text {mat }}=2 \times 10^{4}$ spectra with the largest mutual difference

$$
D_{i j}=\int d E\left|P_{i}(E)-P_{j}(E)\right| .
$$

For each member of this subset of reference spectra, we calculate $n_{\text {pul }}=500$ fluctuating spectra from noisy pulses generated with the partial-coherence method $[11,13]$ with a different noise realization for each (synthetic) Hamilton matrix. Hence, we must propagate about $n_{\text {mat }} \times n_{\text {pul }}=10^{7}$ TDSEs, which takes, however, only a few seconds for a single TDSE thanks to our highly optimized propagation scheme [13]. It includes prediagonalization of the Hamilton matrices which saves computing time since one and the same system is propagated for different pulse realizations. Finally, we have for each Hamilton matrix (1) one reference spectrum $P_{k}^{\text {ref }}(E)$ and $n_{\text {pul }}$ noisy spectra $P_{k l}(E)$, i. e., a total of $n_{\text {mat }} \times\left[n_{\text {pul }}+1\right]$ spectra.

Instead of the individual $P_{k l}(E)$ we use partially averaged spectra $\bar{P}_{k j}(E)=(1 / m) \sum_{l \in\left\{s_{j}\right\}} P_{k l}(E)$ for efficient training. To this end we draw a random subset $\left\{s_{j}\right\}$ containing $m$ spectra from the $n_{\text {pul }}$ fluctuating spectra for each SHM and repeat this procedure $\bar{n}_{\text {pul }}$ times to create $j=1, \ldots, \bar{n}_{\text {pul }}$ averaged spectra. For our application $\bar{n}_{\text {pul }}=$ 10 and $m=200$ is a good compromise between rugged spectra for smaller $m$ and an increased training effort for larger $m$. All spectra are normalized, i.e., $\int d E P(E)=1$.

(v) To complete the final step, the parametrization of the spectra for training, we represent the resulting averaged spectra $\bar{P}_{k j}(E)$ in terms of harmonic oscillator eigenfunctions $\chi_{\kappa}$ as

$$
\bar{P}_{k j}(E)=\left|\sum_{\kappa=1}^{n_{\text {bas }}} \bar{C}_{k j}^{\kappa} \chi_{\kappa-1}(E)\right|^{2},
$$

with the set $\overline{\mathbf{C}} \equiv\left\{\bar{C}_{1}, \ldots, \bar{C}_{n_{\text {bas }}}\right\}$ of coefficients. A basis size of $n_{\text {bas }}=60$ was necessary for the averaged fluctuating spectra, while using a similar expression for the noise-free spectra $n_{\text {bas }}=40$ was sufficient [13]. The network consists of mapping the coefficients $\left\{\overline{\mathbf{C}}_{k j}\right\} \rightarrow\left\{\mathbf{C}_{k j}\right\}$. The training aims at minimizing the difference between the predicted $\mathbf{C}_{k j}$ for the noise-free spectrum and the expected reference spectra $\mathbf{C}_{k}^{\text {ref }}$.

The connection between Hamilton matrices, pulses, and electron spectra just outlined is summarized schematically in Fig. 2.

Building and training the network.-With $n_{\text {mat }}=2 \times 10^{4}$ reference spectra and $\bar{n}_{\text {pul }}=10$ averaged noisy "copies" of each reference spectrum, we have $n \equiv n_{\text {mat }} \times \bar{n}_{\text {pul }}=2 \times 10^{5}$ pairs available for building the network model. Each pair 


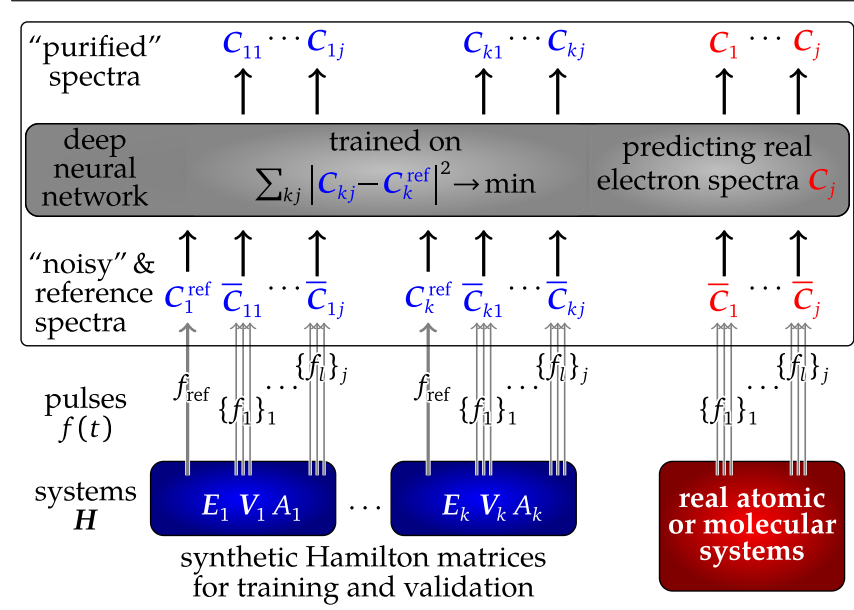

FIG. 2. Building a network with synthetic Hamilton matrices and noisy pulses (left-hand side, blue) and using it (right-hand side, red). The setup of $n_{\text {mat }}$ SHMs is exemplified with the first $\left(\mathbf{H}_{1}\right)$ and $k$ th $\left(\mathbf{H}_{k}\right)$ one. With noisy pulse shapes $f_{l}(t)$ or the reference pulse shape $f_{\text {ref }}(t)$ the SHMs are amended to $\mathbf{H}_{1 l}$ and $\mathbf{H}_{k l}$, respectively, see Eqs. (1). Noisy spectra as calculated with the $\left\{\mathbf{H}_{k l}\right\}$ are averaged over 200 realizations with the same $k$ resulting for each $k=1, \ldots, n_{\text {mat }}$ SHM in ten different spectra with coefficients $\overline{\mathbf{C}}_{k j}, j=1, \ldots, 10$. The network is trained on the predictions $\mathbf{C}_{k j}$ together with the reference $\mathbf{C}_{k}^{\text {ref }}$, i.e., only spectra are processed by the network as emphasized by the black frame. The right-hand side (red) sketches how noisy spectra (from either experiment or theory) to be purified are also averaged over 200 realizations before fed into the trained network to retrieve the reference spectrum.

consists of an averaged noisy spectrum with its respective reference spectrum. Note that the network operates exclusively on the electron spectra, cf. Fig. 2. Once trained, it is therefore directly applicable to the experiment which has only access to spectra.

The full data set with $n$ pairs of spectra is split in the ratio $8: 1: 1$ between training $\left(n_{\text {train }}=0.8 n\right)$, validation $\left(n_{\mathrm{val}}=0.1 n\right)$, and test $\left(n_{\text {test }}=0.1 n\right)$ data. Implemented with the deep-learning library KERAS [27], a fully connected feed-forward neural network is used [13]. The training success and resulting performance of the network as a function of the size of the training data is quantified with the cost function $\delta$, using the basis representation (3) of the spectra, and a more intuitive error $\varepsilon$

$$
\begin{aligned}
\delta_{\Omega} & \equiv \frac{1}{n_{\Omega}} \sum_{j, k=1}^{n_{\Omega}}\left[\mathbf{C}_{k j}-\mathbf{C}_{k}^{\mathrm{ref}}\right]^{2}, \\
\varepsilon_{\Omega} & \equiv \frac{1}{n_{\Omega}} \sum_{j, k=1}^{n_{\Omega}} D_{k j, k \mathrm{ref}},
\end{aligned}
$$

for training ( $\Omega=$ train), validation $(\Omega=$ val), and test ( $\Omega=$ test) data set, respectively. The error $\varepsilon_{\Omega}$ with an upper limit $\varepsilon \leq 2$ measures the difference $D_{k j, k \text { ref }}$, see Eq. (2), between a predicted spectrum $P_{k j}(E)$ and its reference spectrum $P_{k}^{\text {ref }}(E)$. The maximal error $\varepsilon=2$ occurs if the two normalized (i.e., unit-area) spectra are completely disjunct. Both errors (4) decay logarithmically as a function of the SHM data size $n$ [13].

Purification of spectra from SHMs.-We are finally in a position to purify noisy spectra and do this first with the $n_{\text {test }}$ SHM-generated spectra the network was not trained on. Typical snapshots of these spectra are shown in Fig. 3(d). To get a realistic picture we have selected spectra, cf. Figs. 3(a)-3(c), which belong to three groups purified with different residual errors in increasing order: Only 1\% of the spectra have a purification error better than the one shown in Fig. 3(a), the prediction in Fig. 3(b) has a median error $\varepsilon=\varepsilon_{50 \%}$ such that half of the spectra have a smaller, and half of them have a larger prediction error. Finally, only $1 \%$ of the purified spectra have a larger error than the one shown in Fig. 3(c). The gray-shaded curves provide the reference spectrum $P_{k}^{\mathrm{ref}}(E)$ in each case. The simple average (from the test-data set for a specific SHM and field intensity) set is shown as a dashed line.

One sees that the purification works quite well, even for a typical "worst case" as in Fig. 3(c), where all peaks including the fine structure, appear at the correct energies, despite the fact that none of the features is contained in the fully averaged spectra $\bar{P}_{k}(E)=\sum_{j} \bar{P}_{k j}(E)$. We also note that spectra of rather different shapes and details of the structure, from a smooth single peak [Fig. 3(a)] over a triple peak [Fig. 3(b)] to a fine-structured multipeak shape

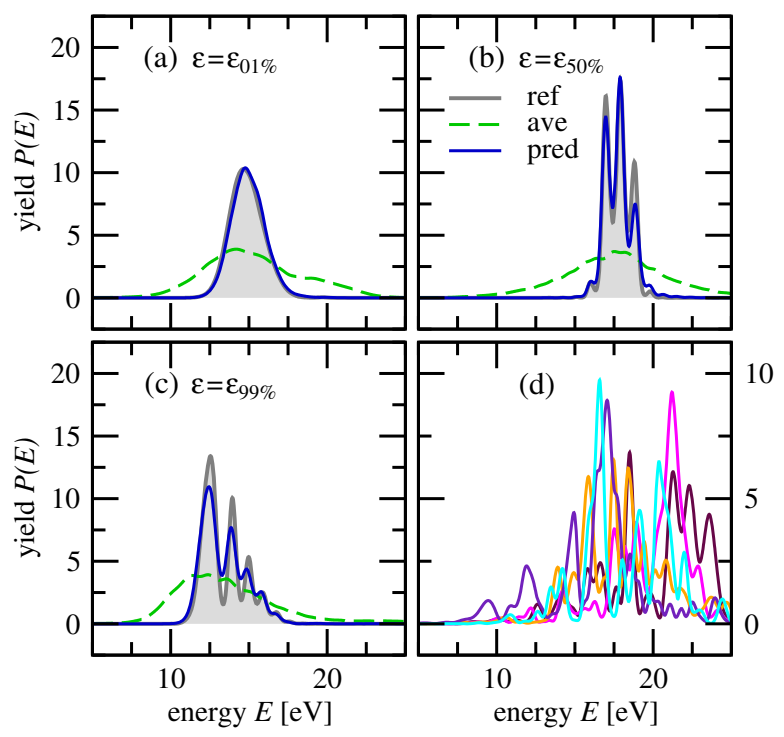

FIG. 3. Photoelectron spectra from the SHM test-data set. The full average of fluctuating spectra (green-dashed line) and prediction from the network (blue) are compared to the reference (gray and shaded). Panels (a)-(c): Examples with three prediction errors $\varepsilon=\varepsilon_{p}$ are shown, with $p$ indicating the percentage of spectra having a smaller error, i.e., $99 \%$ of all spectra from the test-data set have a smaller prediction error than the one shown in panel (c). Panel (d): Five single-shot spectra for the Hamilton matrix used in panel (b). 

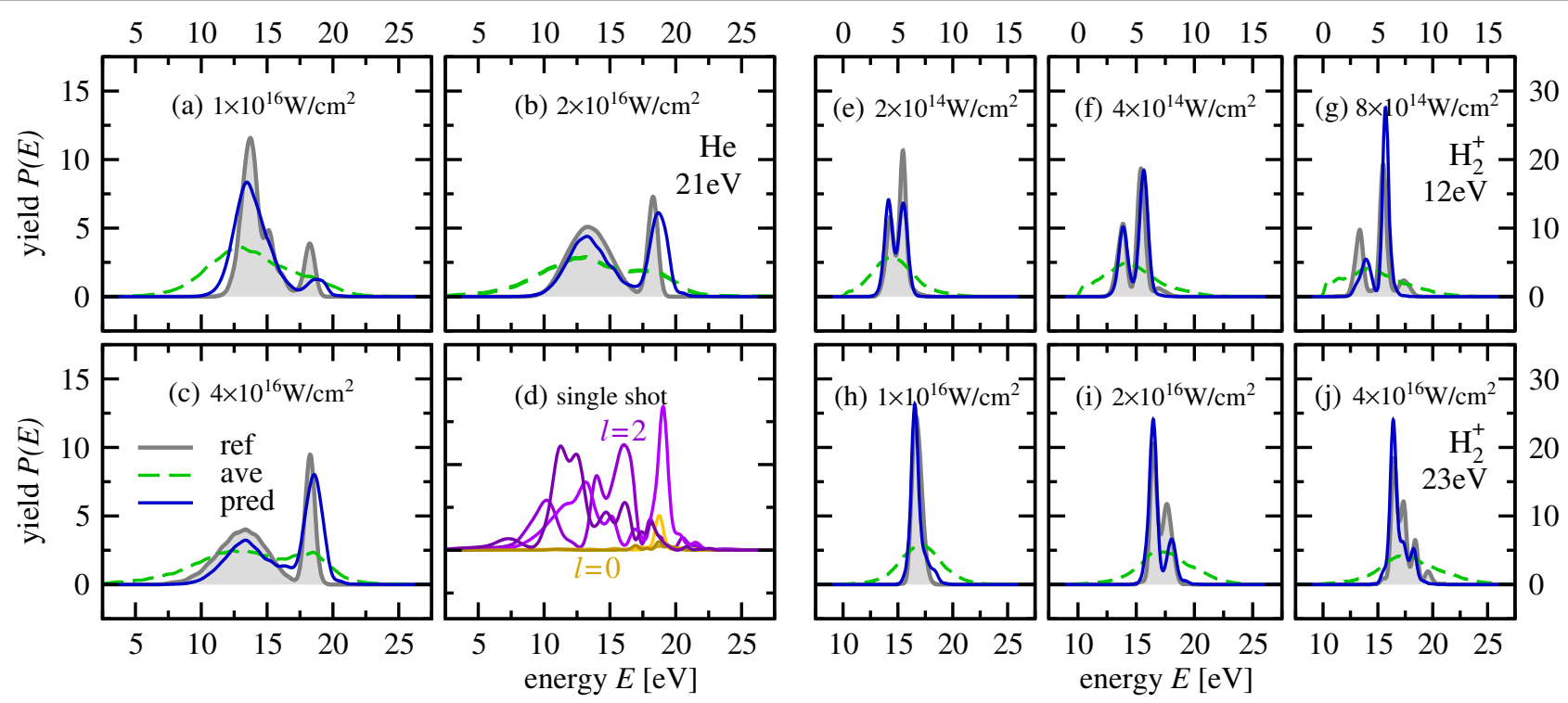

FIG. 4. Photoelectron spectra for the He atom (a)-(d) and the $\mathrm{H}_{2}{ }^{+}$molecule (e)-(j). For He we show results for one photon-frequency $\omega$ (case $\alpha$ in the text), for $\mathrm{H}_{2}{ }^{+}$for two different $\omega$ (cases $\boldsymbol{\beta}$ and $\boldsymbol{\gamma}$ in the text), whereby each combination is presented for three different intensities $I$ (with $\omega$ and $I$ are being specified in the panels). As in Fig. 3, fully averaged and predicted spectra are compared to the reference spectra. Three single-shot spectra for $I=2 \times 10^{16} \mathrm{~W} / \mathrm{cm}^{2}$, as used in panel (b), are shown in panel (d), separately for the $s$ channel ( $\ell=0$, orangelike lines) and the $d$ channel $(\ell=2$, violetlike lines), respectively.

[Fig. 3(c)], can be purified successfully. The rather diverse spectra $P_{k l}(E)$ from fluctuating pulses $f_{l}(t)$ for a specific SHM, as shown in Fig. 3(d), indicate the strong sensitivity to the pulse profile which is due to Stark shifts and AutlerTownes splittings. The complete failure of the fully averaged spectra in revealing the reference spectrum $P_{k}^{\text {ref }}(E)$ is striking. This happens despite the fact that the reference pulse is retrieved by averaging a sufficient number of fluctuating pulses if created by the partialcoherence method [11]. The corresponding reference spectrum, however, is never obtained by averaging the fluctuating spectra since the underlying ionization dynamics is nonlinear. The consequence is an intricate mapping between fluctuating spectra and the reference spectrum, which is constructed with the deep neural network.

Purification of spectra from physical systems.-So far the successful purification referred to spectra not known to the network, but generated through SHMs which were also used to train the network, only with different parameters. In the following, we will apply the trained network to photoelectron spectra for three cases of two different physical systems: $(\boldsymbol{\alpha})$ The He atom dominated by 2-photon absorption [28] and the hydrogen molecule ion $\mathrm{H}_{2}{ }^{+}$ionized by $(\boldsymbol{\beta})$ 2 - and $(\gamma) 3$-photon processes. These spectra have been obtained in full 3D; for technical details see [13]. In case $\boldsymbol{\alpha}$ [Figs. 4(a)-4(c)] the spectra consist of contributions from the $s$ and $d$ manifolds, which can be reached by a 2-photon process, whereby the $d$ channel clearly dominates [cf. Fig. 4(d)]. For $\mathrm{H}_{2}{ }^{+}$, aligned along the laser polarization, either the gerade continuum for case $\boldsymbol{\beta}$ [Figs. 4(h)-4(j)], or the ungerade continuum for case $\gamma$
[Figs. 4(e)-4(g)], is considered. The central frequencies for the laser pulse are chosen according to the transition energies $\omega_{\alpha}=E_{2 p}-E_{1 s}=20.95 \mathrm{eV}, \omega_{\beta}=E_{2 \sigma_{u}}-E_{1 \sigma_{g}}=23.05 \mathrm{eV}$, and $\omega_{\gamma}=E_{1 \sigma_{u}}-E_{1 \sigma_{g}}=11.83 \mathrm{eV}$, respectively. Fluctuating pulses $f_{l}(t)$ are created as before but we use new random realizations. As in the training procedure, we have created ten averaged spectra, which are fed into the trained network. Each one is composed of 200 fluctuating spectra [26]. The ten resulting purified spectra from the network are again averaged to arrive at the network's estimate of the reference spectrum.

We show in Fig. 4 results for three different intensities in the range where few-photon ionization is nonperturbative. As expected from SHM-generated spectra in Fig. 3, the fully averaged spectra (green-dashed lines) do not provide sensible information about the reference spectra. The mapping with the network (blue-solid lines), however, reveals the respective peak structure of the photoelectron spectra.

Note that the network was neither trained on the spectra of the 3D helium atom nor on those of the hydrogen molecule ion, nevertheless these spectra are purified successfully with the network mapping as shown in Fig. 4. The training of the network was performed with synthetic data derived from a representative 1D photoionization dynamics only, which allowed us to keep the size of the Hamilton matrices small enough to be able to compute the $10^{7}$ TDSEs for a sufficient amount of training data. Apparently, although generated from the 1D derived ones, the SHMs represent dynamical systems sufficiently generic such that also realistic 3D spectra from the three rather different processes $\boldsymbol{\alpha}, \boldsymbol{\beta}$, and $\boldsymbol{\gamma}$ could be purified with the 
same network. Hence, it should also work on experimental spectra, which will be slightly different to the extent to which many-electron effects show up in photoelectron spectra as compared to the present $3 \mathrm{D}$ single-active-electron calculations. To measure reference spectra in a proof-ofprinciple experiment one could either use seeded FEL pulses [29-32] or set up an experiment at a coherent (highharmonic) source and generate noisy pulses artificially.

To summarize, we have devised a strategy to purify noisy photoelectron spectra, typical for SASE FELs with the help of a deep neural network. While this example was chosen on purpose to be specific, through its design our approach is far more general. First, we have checked [13] that other noise models [33,34] can be used. Second, purification could be conditioned on any arbitrary reference pulse. Third, and most importantly, the systematic introduction of synthetic Hamilton matrices permits one to generate a training data set of ample size with reasonable computational effort and renders the trained network applicable for scenarios where it was not trained for. In the present example, we applied the network trained on synthetic dynamics to purify realistic 3D spectra. For future work, we would like to point out that noisy pulses driving nonlinear processes are actually advantageous, since they allow one to obtain the target response over a wide spectral and dynamic range in a single shot, provided one has tools to analyze the resulting spectra.

This work has been supported by the Deutsche Forschungsgemeinschaft (DFG) through the priority program 1840 "Quantum Dynamics in Tailored Intense Fields" and "BiGmax", the Max Planck Society's research network on big-data-driven materials science.

[1] V. Dunjko and H. J. Briegel, Machine learning \& artificial intelligence in the quantum domain: A review of recent progress, Rep. Prog. Phys. 81, 074001 (2018).

[2] P. Mehta, M. Bukov, C.-H. Wang, A. G. R. Day, C. Richardson, C. K. Fisher, and D. J. Schwab, A high-bias, low-variance introduction to machine learning for physicists, Phys. Rep. 810, 1 (2019).

[3] G. Carleo, I. Cirac, K. Cranmer, L. Daudet, M. Schuld, N. Tishby, L. Vogt-Maranto, and L. Zdeborová, Machine learning and the physical sciences, Rev. Mod. Phys. 91, 045002 (2019).

[4] G. Carleo and M. Troyer, Solving the quantum many-body problem with artificial neural networks, Science 355, 602 (2017).

[5] A. Sanchez-Gonzalez et al., Accurate prediction of x-ray pulse properties from a free-electron laser using machine learning, Nat. Commun. 8, 15461 (2017).

[6] D. Rogus and M. Lewenstein, Resonant ionisation by smooth laser pulses, J. Phys. B 19, 3051 (1986).

[7] S. H. Autler and C. H. Townes, Stark effect in rapidly varying fields, Phys. Rev. 100, 703 (1955).
[8] C. Meier and V. Engel, Interference Structure in the Photoelectron Spectra Obtained from Multiphoton Ionization of $\mathrm{Na}_{2}$ with a Strong Femtosecond Laser Pulse, Phys. Rev. Lett. 73, 3207 (1994).

[9] P. V. Demekhin and L. S. Cederbaum, Coherent intense resonant laser pulses lead to interference in the time domain seen in the spectrum of the emitted particles, Phys. Rev. A 86, 063412 (2012).

[10] M. Baghery, U. Saalmann, and J. M. Rost, Essential Conditions for Dynamic Interference, Phys. Rev. Lett. 118, 143202 (2017).

[11] T. Pfeifer, Y. Jiang, S. Düsterer, R. Moshammer, and J. Ullrich, Partial-coherence method to model experimental free-electron laser pulse statistics, Opt. Lett. 35, 3441 (2010).

[12] R. Moshammer et al., Second-order autocorrelation of XUV FEL pulses via time-resolved two-photon single ionization of He, Opt. Express 19, 21698 (2011).

[13] See Supplemental Material at http://link.aps.org/ supplemental/10.1103/PhysRevLett.124.113201 for details on generating laser pulses, propagating the time-dependent Schrödinger equation and training of artificial neural networks, which includes additional Refs. [14-21].

[14] G. Geloni, E. Saldin, L. Samoylova, E. Schneidmiller, H. Sinn, T. Tschentscher, and M. Yurkov, Coherence properties of the European XFEL, New J. Phys. 12, 035021 (2010).

[15] X. M. Tong and C. D. Lin, Empirical formula for static field ionization rates of atoms and molecules by lasers in the barrier-suppression regime, J. Phys. B 38, 2593 (2005).

[16] L. D. Landau and E. M. Lifschitz, Quantum Mechanics (Pergamon Press, Oxford, 1989).

[17] X. Glorot, A. Bordes, and Y. Bengio, Deep sparse rectifier neural networks, Proc. Mach. Learn. Res. 15, 315 (2011), http://proceedings.mlr.press/v15/glorot11a.html.

[18] X. Glorot and Y. Bengio, Understanding the difficulty of training deep feedforward neural networks, Proc. Mach. Learn. Res. 9, 249 (2010), http://proceedings.mlr.press/v9/ glorot10a.html.

[19] D. P. Kingma and J. L. Ba, ADAM: A method for stochastic optimization, arXiv:1412.6980.

[20] M. Li, T. Zhang, Y. Chen, and A. J. Smola, Efficient minibatch training for stochastic optimization, in Proceedings of the 20th ACM SIGKDD Conference (Association for Computing Machinery, New York, 2014), p. 661.

[21] N. Srivastava, G. Hinton, A. Krizhevsky, I. Sutskever, and R. Salakhutdinov, Dropout: A simple way to prevent neural networks from overfitting, J. Mach. Learn. Res. 15, 1929 (2014), http://jmlr.org/papers/v15/srivastava14a.html.

[22] K. Tiedtke et al., Gas detectors for x-ray lasers, J. Appl. Phys. 103, 094511 (2008).

[23] L. Tao and A. Scrinzi, Photo-electron momentum spectra from minimal volumes: The time-dependent surface flux method, New J. Phys. 14, 013021 (2012); A. Scrinzi, t-SURFF: Fully differential two-electron photo-emission spectra, New J. Phys. 14, 085008 (2012).

[24] V. Mosert and D. Bauer, Photoelectron spectra with Qprop and t-SURFF, Comput. Phys. Commun. 207, 452 (2016).

[25] S.PatchkovskiiandH. G.Muller,Simple, accurate, andefficient implementation of 1-electron atomic time-dependent 
Schrödinger equation in spherical coordinates, Comput. Phys. Commun. 199, 153(2016).

[26] We will use the terms "reference spectrum" and "fluctuating spectrum" to refer to a "spectrum from a reference pulse" and a "spectrum from a fluctuating pulse", respectively. We never deal with the spectral representation of the pulse itself.

[27] F. Chollet, Keras: The Python deep learning library, https://keras.io (2015).

[28] U. Saalmann, S. K. Giri, and J. M. Rost, Adiabatic Passage to the Continuum: Controlling Ionization with Chirped Laser Pulses, Phys. Rev. Lett. 121, 153203 (2018).

[29] J. Amann et al., Demonstration of self-seeding in a hard-x-ray free-electron laser, Nat. Photonics 6, 693 (2012).
[30] E. Allaria et al., Highly coherent and stable pulses from the FERMI seeded free-electron laser in the extreme ultraviolet, Nat. Photonics 6, 699 (2012).

[31] E. Allaria et al., Two-stage seeded soft-x-ray free-electron laser, Nat. Photonics 7, 913 (2013).

[32] P. R. Ribič et al., Coherent soft x-ray pulses from an echo-enabled harmonic generation free-electron laser, Nat. Photonics 13, 555 (2019).

[33] N. Rohringer and R. Santra, X-ray nonlinear optical processes using a self-amplified spontaneous emission free-electron laser, Phys. Rev. A 76, 033416 (2007).

[34] G. M. Nikolopoulos and P. Lambropoulos, Effects of free-electron-laser field fluctuations on the frequency response of driven atomic resonances, Phys. Rev. A 86, 033420 (2012). 